$\begin{array}{ll}\text { Italique } & \text { Italique } \\ \text { Poésie italienne de la Renaissance }\end{array}$

III | 2000

Varia

\title{
I cinquanta componimenti funebri di Michelangelo per Luigi del Riccio
}

\section{Franz Voelker}

\section{(2) OpenEdition}

\section{Journals}

\section{Edizione digitale}

URL: http://journals.openedition.org/italique/185

DOI: $10.4000 /$ italique.185

ISSN: 1663-4438

\section{Editore}

Librairie Droz

\section{Edizione cartacea}

Data di pubblicazione: 31 dicembre 2000

Paginazione: 23-44

ISBN: 2-600-00490-4

ISSN: 1423-3983

Notizia bibliografica digitale

Franz Voelker, «I cinquanta componimenti funebri di Michelangelo per Luigi del Riccio », Italique [Online], III | 2000, online dal 06 octobre 2009, consultato il 02 mai 2019. URL : http:// journals.openedition.org/italique/185; DOI : 10.4000/italique.185 
FRANZ VOELKER

I C I N Q U A N T A

C O M P O N I E N T I F U N E B R I

D I M C HELA N GELO

PER L U I G I D E L R I C C I O 


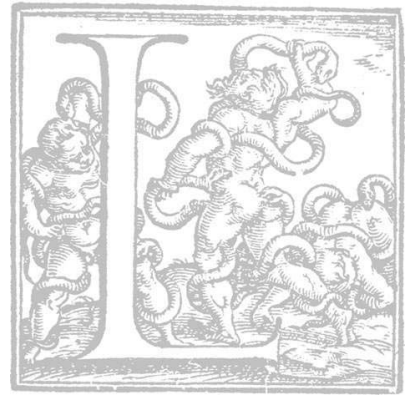

E cinquanta poesie oggetto del presente saggio (Rime $179-228)^{1}$ sono state scritte da Michelangelo a Roma nel I544, per la morte, all'età di quindici anni, di Francesco Bracci, detto Cecchino. Il giovane dovette colpire per la sua bellezza più d'un cultore dell'amore omosessuale nella cerchia di Fiorentini frequentata dal Buonarroti. ${ }^{2}$

I componimenti constano di un madrigale (192), di un sonetto (193) e di quarantotto quartine in endecasillabi a rime incrociate $(A B B A)$. Le quartine tendono allo statuto di epitaffi epigrammatici. Si possono definire epitaffi dato che, a caratterizzarle, sono tre elementi tipici delle iscrizioni tombali : l" identità del defunto", l" ancoraggio topologico ", la " modalità d'esistenza". ${ }^{3}$ La realizzazione di questi tre elementi può variare da testo a testo. Citerò ad esempio questi due tetrastici :

Qui giace il Braccio, e men non si desìa sepulcro al corpo, a l'alma il sacro ufizio. Se più che vivo, morto ha degno ospizio in terra e 'n ciel, morte gli è dolce e pia.

Se qui son chiusi i begli occhi e sepolti anzi tempo, sol questo ne conforta : che pietà di lor vivi era qua morta ; or che son morti, di lor vive in molti.

(179)

Il nome "Braccio » e la sineddoche « $i$ begli occhi $》$ rivelano l" identità del defunto", il deittico "qui » fornisce un "ancoraggio topologico" a questa identità e i verbi " giace", "son chiusi e sepolti » specificano la "modalità d'esistenza" di chi è nella tomba additata dal deittico. Importa precisare che le quartine di Michelangelo costituiscono degli epitaffi meramente letterari, sono epitaffi fittizi. Lo scarto che esiste fra questi tetrastici e la pratica epigrafica rinascimentale non consente affatto di immaginare che siano stati ideati con l'intento di essere effettivamente incisi nella pietra. ${ }^{4}$

Oltreché epitaffi, numerose quartine possono anche definirsi epigrammi. Esse corrispondono, in certi casi in modo più evidente che in altri, a una definizione che Giulio Cesare Scaligero dà di questo genere nella sua Poetica (Poetices 
libri septem, I ed. Lione, Is61, libro III, capitolo I25). Secondo lo Scaligero, un epigramma è una poesia breve che si compone di due parti : la prima contiene l'indicazione di una cosa, di una persona o di un'azione, mentre la seconda "deduce» con un tratto di spirito, con un'arguzia (il termine "dedurre" ha qui un senso generale e impreciso) qualcosa a partire dalle premesse che questa indicazione fornisce. "Il trattato Agudeza y arte de ingenio di Baltasar Gracián (I ed. Madrid, I642 ; ed. definitiva Huesca, I648) puì servire da complemento all'analisi scaligeriana, in quanto offre un esame e una tipologia di vari tipi d'arguzia. ${ }^{6}$

Per facilitare la percezione dell" "epigrammaticità" delle quartine di Michelangelo, conviene pure tener presente l'analisi che Gotthold Ephraim Lessing presenta nelle sue Zerstreute Anmerkungen über das Epigramm und einige der vornehmsten Epigrammatisten (I ed. Berlino, I77I). Anche Lessing evidenzia un ritmo binario dell'epigramma, un ritmo però di natura psicologica, anziché logica come nello Scaligero. La prima parte del componimento ha la funzione di destare la curiosità del lettore e la seconda soddisfa questa curiosità. L'epigramma suppone dunque nel lettore la successione di due affetti : in un primo tempo viene creata un'attesa, un'aspettativa ("Erwartung»), ossia una tensione risolta poi dalla conclusione del testo ("Aufschluss 》, cioè letteralmente "spiegazione", "chiarimento"). ${ }^{7}$

Applicando alla quartina 179 le definizioni di Scaligero e di Lessing, si può osservare sia una bipartizione del testo (2+2), sia il fatto che i versi di chiusa formano un'arguzia tramite un gioco di antitesi e mettono fine all'attesa suscitata dalle parole "sol questo ne conforta» (v. 2). La quartina 201 si divide invece in $3+I$ e il verso finale viene a spiegare con spirito ("nacqui ove la morte muore ») perché Cecchino teme "il ritornare in vita 》 (v. 2).

Va infine ricordato che Scaligero, Gracián e Lessing formulano nelle loro teorizzazioni dei principi che reggono una pratica di scrittura ben anteriore. Le loro riflessioni hanno infatti come punto di riferimento soprattutto testi dell' antichità e sono particolarmente valide per Marziale, ${ }^{8}$ la cui influenza è stata cospicua nell' ambito della rinascita dell'epigramma verificatasi in Italia alla fine del Quattrocento. 9

Destinatario delle cinquanta poesie era un amico di Michelangelo, Luigi del Riccio (? - I546), fuoriuscito fiorentino che viveva da anni a Roma. ${ }^{10}$ Questo parente di Cecchino riceveva $i$ testi del Buonarroti man mano che venivano composti. Un terzo circa dei componimenti era accompagnato da annotazioni di Michelangelo che si leggono tuttora negli autografi. ${ }^{\text {"I }}$ Il loro tenore è vario. Certe sono commenti alla scrittura, altre contengono la menzione di cibi. S'incontra ad esempio : 
I COMPONIMENTI FUNEBRI DI MICHELANGELO

Chi qui morto mi piange indarno spera, bagniando l'ossa e 'l mie sepulcro, tucto ritornarmi com'arbor secho al fructo; c'uon morto non risurge a primauera.

Questo goffo decto mille nolte pe' finochi

(199).

I' temo più, fuor degli anni e dell'ore che m'àn qui chiuso, il ritornare in uita, s'esser può qua, ch' i' non fe' la partita ; po' c'allor naqqui oue la morte muore.

Questo dicono le trote e non io; però, s' e' uersi non ui piacciono, non le marinate più senza pepe.

$(20 \mathrm{I})$

In altre annotazioni, Michelangelo propone delle varianti al testo. Ne citerò una notevole per la forte ambiguità carnale: ${ }^{\mathrm{I} 2}$

La carne terra, e qui l'ossa, già prive de' lor begli ochi e del leggiadro aspecto, fan fede a quel ch' i' fu' gratia e dilecto in che carcer quagg[i]ù l'anima uiue.

Pigliate questi dua uersi di socto, che son cosa morale; e questo ui mando per la recta de' quindici polizini. fan fede a quel ch' i' fu' gratia nel lecto, che abbracc[i]aua e 'n che l'anima uiue.

(197)

Il corpus di testi per Cecchino può suscitare vari interrogativi. In queste pagine tenterò di chiarire perché Michelangelo ha scelto il genere dell'epitaffio epigrammatico e perché ha scritto ben quarantotto epitaffi per lo stesso defunto. Proverò inoltre a dare ragione della funzione dei poscritti, il cui tono si rivela non di rado assai poco funebre.

\section{Una collettanea per la morte di Cecchino Bracci}

Un esame dei manoscritti consente di collocare le poesie di Michelangelo in un contesto affine, sottraendole alla loro apparente singolarità. Sono infatti incluse 
in una collettanea in morte, un genere in gran voga nel Cinquecento. Il Riccio le ha ricopiate in un piccolo quaderno (incluso ora nel Codice Magliabechiano VIII. 38$)^{13}$ prive dei poscritti e assieme a dodici componimenti - sonetti e madrigali per lo più-scritti da sei altri autori per la morte di Cecchino. Su un totale complessivo di 332 versi, 220, ossia circa due terzi, sono del Buonarroti. Con la sua produzione estesa, Michelangelo ba dato opera più di ogni altro al monumento poetico destinato a onorare il giovane defunto. Egli ha in tal modo corrisposto al desiderio espresso da Luigi del Riccio di ottenere molti testi per Cecchino (cfr. $i$ commenti in calce a 190, 198, 2II, 2I4), un desiderio che si confaceva alla probabile volontà del Buonarroti di rendere omaggio al morto. Inoltre, e ciò spiegherebbe pure un contributo così schiacciante, la collettanea ha forse rappresentato per Michelangelo l'occasione per affermarsi come rimatore: un banco di prova insperato per misurarsi con gli altri partecipanti, fra i quali letterati provetti come Anton Francesco Grazzini, Donato Giannotti e Paolo del Rosso. Una raccolta in morte è per sua natura una sorta di concorso poetico, poiché ogni partecipante è chiamato a trattare lo stesso argomento $e$ perciò a confrontarsi con gli altri contributori su un terreno comune. ${ }^{14}$

Attraverso il dispositivo formato dai componimenti 193-194, Michelangelo manifesta forse il suo intento di imporsi nel settore della poesia, un intento che il Riccio pare assecondare, quando ad esempio provvede con numerose copie alla diffusione dei testi di lui. ${ }^{15}$ Il sonetto 193 esprime il rifunto di fare un ritratto del giovane defunto e rinvia al successivo epitaffio tramite un verso identico (193, I2 = 194, 4: "se l'un ne l'altro amante si trasforma »), come se il Buonarroti respingesse il ruolo di scultore, di pittore, per rivendicare quello di poeta. ${ }^{16}$ Va ricordato che attorno al I544-I546 Michelangelo dimostra un interesse particolare per la propria produzione in versi. In quel periodo egli aduna infatti, con l'aiuto di Luigi del Riccio e Donato Giannotti, le poesie fino allora da lui composte e ne costituisce una raccolta. ${ }^{17}$ A parer mio, la formazione di una tale silloge denota in Michelangelo il desiderio di affermare, almeno materialmente, la propria figura di rimatore.

Gli anni posteriori al IS44 saranno ricchi di omaggi resi alla poesia di Michelangelo. Del I546 circa è il dialogo di Donato Giannotti in cui Michelangelo è messo in scena e onorato non solo come grande conoscitore di Dante, ma anche come poeta, dato che ciascuna delle due parti della discussione si chiude sulla recitazione da parte del Buonarroti di una sua lirica. ${ }^{18} \mathrm{La}$ famosa Lezione in cui Benedetto Varchi commenta il sonetto di Michelangelo Non ha l'ottimo artista alcun concetto (Rime ISI) e cita vari altri componimenti del Maestro, ha luogo nel I546. Il testo ne sarà poi pubblicato a Firenze nel I549. ${ }^{19}$ Il Vasari, dal canto suo, includerà una poesia di Michelangelo nell'edizione delle Vite uscita nel I550. E la sua scelta, importa sottolinearlo, cade non già su un sonetto o un madrigale, ma su una quartina 
epigrammatica (Caro m'è 'l sonno, e più l'esser di sasso, Rime 247), risposta vincente di Michelangelo alla quartina di un altro autore, Giovanni di Carlo Strozzi (La Notte che tu vedi in sì dolci atti), parimenti citata. ${ }^{20}$

La scelta dell'epitaffio epigrammatico in forma di quartina si rivela particolarmente adeguata per il doppio scopo di onorare Cecchino e affermarsi come rimatore. Dato il suo breve respiro, la quartina rende possibile la redazione di numerosi componimenti. A favorire la produzione di molti testi è pure il carattere "formulare" del genere dell'epitaffio epigrammatico. La realizzazione dei tratti definitori di cui si è detto, ricorrenti di poesia in poesia, permette di praticare una scrittura a base di variazioni su un certo numero di parametri fissi, cioè una scrittura facilmente copiosa. ${ }^{21}$ Con questo gioco di variazioni, il Buonarroti può inoltre evidenziare la sua abilità, la sua virtuosità, dimostrandosi capace di comporre ben quarantotto epitaffi, simili e diversi insieme, per lo stesso defunto. Infine, il ricorso a un genere "brillante" come l'epigramma, fondato sull'uso di arguzie, trova forse una spiegazione nel contesto agonistico della collettanea.

Optando per l'epitaffio epigrammatico in quartine a rime incrociate, Michelangelo adopera un tipo di testo in voga a quella data. Questa sorta di produzione si afferma nel corso della prima metà del Cinquecento ${ }^{22} e$ il quarto decennio del secolo ne vede una vera e propria fioritura, del tutto coincidente col breve periodo in cui il Buonarroti ne fa uso. Benché l'attività poetica di Michelangelo si estenda sull'arco di una sessantina d'anni (circa dal I503 al I560), oltre ai tetrastici per il Bracci (I544), si dà solo un altro caso d'impiego dell'epitaffio in quartina, che risale all'incirca al I543 (Rime 177 ). Ascrivibile a questi anni quaranta è una serie di quindici quartine d'omaggio a Dante, fra le quali si annoverano degli epitaffi epigrammatici. ${ }^{23} \mathrm{Da}$ considerarsi come una delle prime prove poetiche dell'Accademia degli Umidi, fondata a Firenze nel novembre IS40, la serie risalirebbe alla fine del I540 o allinizio del I54I. ${ }^{24}$ Tra i diversi accademici che vi diedero mano spicca la figura del Fiorentino Anton Francesco Grazzini, detto il Lasca (I504-1584). Membro fondatore dell'Accademia degli Umidi, contributore alla collettanea per Cecchino, egli è inoltre l'autore di un certo numero di epitaffi in quartine di registro satirico-burlesco. ${ }^{25}$ Il Grazzini, in virtù di questa sua abilità in più prove, potrebbe aver avuto un ruolo nell'avviare Michelangelo al genere dell'epitaffio epigrammatico.

Rispettivamente del IISI-IS42 e del I544 sono due pasquinate romane costituite ciascuna da una serie di epitaffi di schema rimico $A B B A .{ }^{26}$ Infine, un insieme di epitaffi epigrammatici redatti in tetrastici si trova nella prima edizione, detta torrentiniana, delle Vite del Vasari (I550), opera in preparazione almeno dal ${ }^{1540 .}{ }^{27}$ I vari testi che ho menzionato condividono con le 
quartine per Cecchino un certo numero di caratteristiche. Tuttavia la redazione di una ampia serie di epitaffi dedicati a uno stesso defunto è una peculiarità esclusivamente buonarrotiana.

Dal punto di vista tematico, la serie di tetrastici scritti da Michelangelo appare essenzialmente come un insieme di variazioni sul tema della consolatio. $\mathrm{Di}$ testo in testo sono rivolti ai vivi, in modo variamente esplicito, argomenti consolatori per la morte del giovane Bracci. In rilievo di rima, la parola "conforta» della quartina che apre la serie (I79) sembra annunciare questa tematica, una tematica singolarmente evidenziata da un certo numero di formule brevi in chiusa di epitaffio: "vive in molti》 (179, 4), "non son morto 》 (190, 4), "resto in te vivo 》 (194, 3), "nacqui ove la morte muore» (20I, 4), " gli è'l morir buono » (204, 4), " morte gli è dolce e pia 》 (22I, 4), ecc. Come la scelta dell'epitaffio epigrammatico in quartina, il ricorso, pressoché esclusivo, ${ }^{28}$ a un solo materiale tematico, che include per di più numerosi luoghi comuni, è del tutto congruo alla duplice finalità perseguita da Michelangelo: onorare il defunto con un contributo massiccio alla silloge che gli viene dedicata, dar prova della propria fecondità, abilità e inventività. Un tema come quello della consolatio apre infatti la possibilità di molteplici giochi di variazione, di differenziazione, tra le stesse quartine buonarrotiane, oppure rispetto alla tradizione o a testi in cui altri partecipanti alla collettanea si servano di luoghi comuni consolatori. È però necessario completare questa prima diagnosi relativa alla funzione della tematica consolatoria e dar rilievo al fatto che le quartine sono state composte da Michelangelo per la morte di un giovane al quale il destinatario dei testi, Luigi del Riccio, era molto legato. Pochi giorni dopo il decesso di Cecchino (avvenuto l'8 gennaio I544), Luigi scriveva a Donato Giannotti : ${ }^{29}$

\footnotetext{
Oyme, messer Donato mio ! Il nostro Cechino è morto ! [...] Tutta Roma lo piagnie. Messer Michelagnolo mi fa il disegnio d'uno onesto sepulcro di marmo, et uoi ui degnierete di fare lo epitaffio et mandarmelo con una epistola confortatoria, se sarà a tempo, che mi ha cauato l'anima. Patientia! Viuo con mille et mille morte l'ora. O Dio! Come fortuna ua cangiando stile! - Il tuo (?) Luigi del Riccio disperato.
}

A tale bisogno di conforto pare rispondere la serie di tetrastici mandati da Michelangelo all'amico. Mentre nei componimenti degli altri contributori alla raccolta in morte prevale il compianto funebre, $i$ versi al centro del madrigale 192 affermano la necessità per $i$ vivi di porre un termine ("preschiver») al proprio dolore e di trasformarlo "in riso», ossia di consolarsi della morte del giovane Bracci, poiché egli (cfr. vv. I-6) è andato a miglior vita : 
Dunche, sine peccata, in riso ogni suo doglia preschiver debbe alcun del suo defunto

(192, 7-9)

\section{Argomenti consolatori}

Riconducibili in gran parte ai luoghi comuni della consolatio classica o cristiana, gli argomenti a cui Michelangelo ricorre possono essere suddivisi in quattro categorie. ${ }^{30}$ Alcuni (I) si fondano sull'idea d'inanità di ogni dolore. Ad esempio, affinché cessi la tristezza eccessiva di chi sopravvive a Cecchino, le lacrime sono tenute per inutili perché la situazione del defunto è migliore di quella dei vivi (I80), oppure perché non possono ridare vita al morto (199). Un'altra sorta di argomenti (II) consiste nel ricordare che la morte, in quanto inerente alla condizione umana, costituisce un evento inevitabile, ba una potenza irresistibile. ${ }^{31}$ Il tetrastico I84 sembra esprimere questo assunto col topos "la lotta contro la morte è inutile": ${ }^{32}$

Qui son de' Bracci, deboli a l'impresa contr'a la morte mia per non morire ; meglio era esser de' piedi per fuggire che de' Bracci e non far da lei difesa

A mio avviso, il luogo comune funebre dà un sapore tragico a un'arguzia (vv. 3-4) che potrebbe suscitare il riso con il suo gioco di parole "Bracci I bracci » e il ravvicinamento sorprendente "Bracci / piedi». Si può ancora aggiungere tra $i$ modi di alludere all'ineluttabilità del decesso la menzione della volontà divina $(213,3-4)$. Ma di gran lunga più numerosi sono, nelle quartine, gli argomenti (III) che fanno della morte un bene. La morte può essere un bene perché libera il defunto dagli incomodi della vita. Viene meno così la tirannia della Fortuna (I80, 4), del Tempo e della Morte (20I). Il morire è anche evento benefico, in quanto ne scaturiscono diversi modi d'immortalità. Immortalità terrena, in primo luogo, poiché una morte prematura nel fiore della bellezza conferisce a Cecchino la vita nel cuore dei vivi, o per la pietà che la sua scomparsa suscita (179, 3-4), o per l'amore che gli viene conservato $(203,4) \mathrm{e}$, anzi, maggiormente riservato $(204,3-4)$. Una vita imperitura è pure conferita quaggiù al defunto dalla tomba in pietra che lo ricorda (2I5, 3-4). L'immortalità vera e propria fa perno essenzialmente sul dualismo "anima-corpo". Liberata dalla prigione corporea grazie alla morte (197, 4), l'anima sale in cielo a vivere meglio (187, 3-4; 223, 3-4; ecc.), e il 
carattere prematuro del decesso agevola questa salita in "paradiso 》 $(219,3)$. Da giovane, Cecchino muore scevro di peccati, mentre se fosse vissuto a lungo, avrebbe corso il rischio di peccare e percio di dannarsi (I81, 3-4; 224, 4, ecc.). ${ }^{33}$ Per concludere questa rassegna, menzionerò ancora il gruppo di argomenti consolatori (Iv) di natura escatologica. La resurrezione del corpo il giorno del Giudizio Universale viene ad esempio menzionata nelle quartine 186,4 e I88, $3-4$.

Molto spesso gli argomenti consolatori sono espressi in forma di arguzia nella seconda parte dell'epigramma. La formulazione "concettosa" può servire a vivificare $i$ luoghi comuni della consolatio, a renderli capaci di "penetrare l'animo ${ }^{34}$ del lettore triste (cfr. I84). Ma tale formulazione possiede anche una valenza consolatoria propria. Quando, ad esempio, l'arguzia consiste nel chiudere il componimento con un argomento consolatorio espresso in forma di sentenza generale (I8I, 3-4; 208, 3-4), la morte prematura di Cecchino (208, I-2) diviene l'effetto di una legge universale che governa il mondo. Un altro tipo d'arguzia (cfr. I79, 3-4) stabilisce una correlazione tra due momenti del destino del Bracci, uno prima e l'altro dopo la sua morte, in modo che la simmetria messa in rilievo manifesti una coerenza, una segreta logica nell'andamento della sorte del giovane, ossia la necessità di tale sorte. ${ }^{35}$ Sia gli argomenti consolatori, sia il meccanismo dell'arguzia concorrono dunque a negare il carattere assurdo della morte prematura di Cecchino, cercando di dominarla con la ragione, di darle un senso, rendendola insomma accettabile. ${ }^{36}$

I rapporti tra arguzia e consolatio possono esser visti anche in un'altra luce. Dato che gli argomenti consolatori "convertono" il decesso del quindicenne Cecchino in un evento sopportabile, essi realizzano l'operazione tipica della specie d'arguzia detta "trasposizione", la quale consiste nel trasformare un evento convertendolo nel contrario di ciò che sembra. ${ }^{37}$ La morte diventa ad esempio «dolce e pia 》 $(221,4)$, il morire «buono 》 $(204,4)$. Ciò significa che la consolatio non è solo un tema dei componimenti, bensi l'origine stessa del carattere epigrammatico di gran parte delle quartine.

Come l'epigramma tramite l'arguzia, il genere dell'epitaffio collabora all'intento consolatorio. Il deittico " qui » che vi ricorre pressoché costantemente addita la tomba, che possiede la funzione di mantenere il defunto tra i vivi e di conservarne il nome. Questa funzione commemorativa è esplicitamente ricordata nelle quartine 200, I-2 e 2I5, 4. Si osservi che a ribadire l'ostentazione del sepolcro per via del deittico sono pure $i$ casi in cui la tomba stessa si esprime al discorso diretto $(212,214,216,218)$ e l'esplicita menzione del monumento funebre (I87, 2 : "questo avello »; I88, 4: "questa sepoltura》, ecc.).

Poiché enuncia la "modalità d'esistenza" di chi è nel sepolcro, l'epitaffio offre ancora a Michelangelo la possibilità di evocare con perentorietà la corruzione 
della salma. Vero è che i termini più frequentemente impiegati per designare lo stato del giovane nella tomba si limitano a indicare la presenza del corpo, e occultano il disfacimento del cadavere (cfr. I80, 2, I87, 2: "esser chiuso »; I8), I, 2I3, I : «esser sepolto; I82, 3, 22I, I : «giacere 》). Questa occultazione sistematica, propria al genere dell'epitaffio, ${ }^{38}$ viene però meno in un certo numero di tetrastici (in particolare nella sequenza 197-199) :

La carne terra, e qui l'ossa mie, prive

de' lor begli occhi e del leggiadro aspetto

$(\mathrm{I} 97, \mathrm{I}-2)$

gli altru' pianti a quest'ossa carne e sangue

bagnando l'ossa e 'l mie sepulcro

$(199,2)$

gli è qui terra or la bellezza mia

$$
(2 \mathrm{II}, 2)
$$

I' fu' de' Bracci, e qui dell'alma privo per esser da beltà fatt' ossa e terra: prego il sasso non s'apra, che mi serra, per restar bello in chi m'amò già vivo.

Il fine di simili notażioni è esplicitato da Michelangelo:

La carne terra, e qui l'ossa mie, prive de' lor begli occhi e del leggiadro aspetto, fan fede a quel ch' i' fu' grazia e diletto in che carcer quaggiù l'anima vive.

Secondo questi versi, il disfacimento della salma dopo la morte (I-2) serve a dimostrare (3: "fan fede 》) la vera natura del corpo, ossia il suo scarso valore. Nell'ambito dell'ideologia dualistica che connota $i$ testi in esame, la perdita di un simile involucro putrescibile, ossia la morte di Cecchino, non può rappresentare che un bene, per il defunto come per chi sopravvive al giovane.

Ma la corruzione di un corpo intimamente conosciuto puo evidenziare in modo ancora più netto quanto fallace era il suo aspetto seducente. Contrapponendo alla miseria della salma il corpo concreto del giovane amante, i versi 3-4 
della variante di 197 accrescono la forza d'edificazione della quartina. Da astratti e generici nella prima redazione (" grazia », " diletto »), i termini per descrivere la fruizione della persona di Cecchino si fanno carnali nella seconda ("lecto », «abbracc[i]aua »):

fan fede a quel ch' i' fu' gratia nel lecto, che abbracc[i]aua e 'n che l'anima uiue.

(197, variante a 3-4)

La qualificazione di «cosa morale » usata da Michelangelo nel commento a questa variante può dunque essere presa alla lettera, senza che questo escluda un suo possibile valore antifrastico.

Che senso attribuire all'accumulazione di argomenti consolatori che si può scorgere nel corpus o, più esattamente, alla ripetizione, con variazione, di quei pochi assunti fondamentali ("la morte è un bene", "la morte è inerente alla condizione umana", ecc.) ai quali sono riconducibili gli argomenti consolatori usati nelle quartine? Si potrebbe supporre che l'effetto ricercato sia la persuasione. Sennonché la relazione tra chi scrive e gli argomenti consolatori è problematica, come risulta dal tetrastico 228, un testo che la sua posizione mette in rilievo. Questa quartina - le parole " non pel numero » nel commento di Michelangelo lo sottolineano - chiude in qualità di cinquantesimo componimento la serie funebre dedicata dal Buonarroti a Cecchino. É cioè la poesia che fa raggiungere al contributo di Michelangelo un numero tondo, ricorrente e privilegiato nella produzione di sillogi durante il Rinascimento. ${ }^{39}$

\section{La presenza umana}

La quartina 228 contesta apertamente la forza consolatoria del luogo comune secondo il quale la vita non è data, ma solo prestata all'uomo, e percio la morte non è che il momento, del tutto normale, della restituzione di questo prestito : ${ }^{40}$

Se 'l mondo il corpo, e l'alma il ciel ne presta per lungo tempo, il morto qui de' Bracci qual salute fie mai che 'l soddisfacci? Di tanti anni e beltà creditor resta.

Per baia e non pel numero. 
Si potrebbe parafrasare cosi : "Se a noi vivi [Michelangelo, Luigi del Riccio, ecc.] il mondo presta il corpo e il Cielo l'anima per un lungo tempo, il giovane Bracci, morto prematuramente, quale salvezza eterna potrà mai renderlo soddisfatto della sua sorte? Egli resta creditore di tanti anni non vissuti e di tanta bellezza perduta". La parola «creditor» (4) segna un doppio rovesciamento rispetto al tòpos : da un lato, l'uomo non appare soltanto come il debitore di Dio per la vita ricevuta, dall'altro, l'esistenza mondana non si trova come al solito svalutata completamente rispetto alla sorte in cielo dopo il decesso. Al contrario di quanto affermano numerose quartine, la morte non è un bene secondo 228, e Cecchino non raggiunge uno stato di pienezza.

Il tenore del tetrastico è commentato dall'autore. L'espressione "per baia», ossia per burla, per scherzo, evidenzia il contenuto poco ortodosso di questi versi, e nello stesso tempo ne limita la portata, come per segnalare che non si tratta di una condanna radicale dell'uso degli argomenti consolatori e dell' ideologia che esprimono. Il fine essenziale di 228 sembra essere quello di attestare che Michelangelo non s'illude sull'efficacia degli argomenti consolatori. La contestazione di un argomento come quello del "prestito", argomento che niente distingue da tanti altri, emblematizza a parer mio l'intrinseca insufficienza dei mezzi razionali usati nell'impresa consolatoria. Qualunque argomento consolatorio può essere contestato perché nessun ragionamento si dimostra veramente capace di consolare chi perde un giovane di quindici anni. E se questi argomenti vengono usati nonostante tutto, $\grave{e}$ in mancanza di meglio. In questa prospettiva, l'accumulazione di argomenti consolatori nel corpus possiede un valore di critica interna. Una simile inflazione conduce alla svalutazione di tali argomenti. ${ }^{4 \mathrm{I}}$

Questa insufficienza dichiarata della ragione nellimpresa consolatoria mi spinge a non prendere $i$ testi di Michelangelo soltanto alla lettera, ma a discernere in essi anche un'operazione consolatoria compiuta con strumenti altri che quelli razionali. Quali strumenti? Si potrebbe dire, in sintonia con alcune riflessioni prerinascimentali sulla consolatio, ${ }^{42}$ che Michelangelo affida l'operazione consolatoria non tanto a ciò che le quartine dicono, ma ben più all'amicizia, alla simpatia, alla presenza umana che questi tetrastici permettono di manifestare al loro destinatario. Scrivendo numerose quartine, non solo il Buonarroti soddisfa il desiderio che Luigi del Riccio aveva di ottenere molti testi, ma anche " accompagna" Luigi nel suo lutto, dato che la composizione dei tetrastici, ricevuti dall'amico man mano che vengono composti, si protrae per mesi. L'impiego di luoghi comuni come quelli della consolatio facilita questo indirizzarsi all'amico addolorato, poiché essi fanno parte di un patrimonio culturale comune al Buonarroti e al Riccio.

A tale idea di presenza umana si può forse ricondurre ciò che definirei la dimensione comica dei testi in esame : si tratta, per Michelangelo, di divertire 
Luigi del Riccio, di ridere assieme a lui. Questo comico, a cui pare alludere il sintagma «in riso » al centro del madrigale 192 (8), sembra nascere da una "mancanza di convenienza e di misura ». ${ }^{43}$ Con questi termini riprendo una formula usata a proposito della poesia burlesca, un genere in cui Michelangelo si è illustrato più volte (cfr. ad esempio Rime 54 ). ${ }^{44}$

La menzione di cibi nei poscritti di vari epitaffi potrebbe costituire una prima sconvenienza capace di suscitare il riso. Si deve tuttavia osservare che Michelangelo era solito ricordare, in calce alle poesie che inviava a mo' di ringraziamento, gli alimenti ricevuti in regalo da conoscenti. Il poscritto al madrigale 143 destinato al Riccio suona ad esempio così (cfr. anche i commenti del Buonarroti alle Rime $145,157,167)$ :

De' melloni e del uino ui ringrazio e pago d'un polizino.

Nell'ambito dei componimenti per Cecchino, $i$ cibi ricevuti dal Buonarroti paiono avere lo statuto di rimunerazione, a immagine di una pratica corrente durante il Rinascimento: il pagamento in natura del salario che spettava a un artista. ${ }^{45}$ Ma nel definire la funzione di questi alimenti, $i$ rapporti affettivi che legavano Michelangelo a Luigi del Riccio e a Cecchino suggeriscono di preferire alla nozione economica di "paga" quella di " mezzo di sollecitazione e di ringraziamento". Che tramite l'invio di cibi Luigi del Riccio manifestasse il suo desiderio di ricevere ancora e ancora dei testi, è ciò che lasciano intendere le parole di Michelangelo in calce alla quartina 190 :

Quande uoi non ne uolete, non mi mandate più niente.

Alla luce di queste osservazioni, il rapporto tra epitaffi e vivande e la menzione di cibi nei poscritti mi paiono perdere molto della loro potenziale comicità. Va però notato che alcune volte il legame tra poesie e cibo è espresso in modo particolare, come nel commento alla quartina 20I, dove l'enunciazione stessa dell'epitaffio è assegnata alle trote mandate da Luigi del Riccio a Michelangelo :

Questo dicono le trote e non io ; però, s' e' uersi non ui piacciono, non le marinate più senza pepe.

Un parallelo fra trote e tetrastico è anzi tratteggiato in questo commento: come già i pesci marinati senza pepe, la quartina potrebbe non piacere. Nel poscritto ai tetrastici 220-223, il rapporto testi-alimenti va fino allidentificazione. Michelangelo vi dice mandare per tre dolci di farina e miele ( $i$ «berri- 
quocoli ») quattro quartine, chiamate da lui « berlingozzi», vale a dire ciambelle dalla crosta croccante:

$$
\text { [...] ui mando quactro berlingozzi pe' tre berriquocoli [...] }
$$

In questi due esempi, la sconvenienza comica pare apertamente ricercata da Michelangelo. Un simile gioco di "degradazione culinaria» ${ }^{46}$ dei componimenti è anche percepibile nella disproporzione espressa dal commento a proposito della quartina 199. Per equivalere agli ortaggi ricevuti, il tetrastico va "moltiplicato" mille volte:

Questo goffo decto mille uolte pe' finochi.

Non è tuttavia escluso che il termine "finochi» sia ambivalente e che si riferisca nello stesso tempo agli ortaggi ricevuti e al contenuto dell'epitaffio. I versi del tetrastico si rivolgono infatti a chi piange Cecchino, ossia ai suoi "mille amanti $(190,2-3):$ "mille amanti » per cui la quartina va detta "mille volte » e che si possono beffardamente chiamare "finochi ${ }^{4}{ }^{47}$ Se questo fosse, la relazione tra quartina e cibo sarebbe molto stretta nel caso di 199. La presenza della sessualità in un contesto funebre sembra costituire un'altra sconvenienza. A questo proposito, conviene ricordare soprattutto la variante del tetrastico r97 citata piu sopra, dove si menzionano «lecto » e " abbracci 》. Il carattere indebito, e perciò comico, di questa menzione è sottolineato dalla qualificazione antifrastica che Michelangelo gli assegna nel poscritto con l'espressione «cosa morale 》. ${ }^{48}$

Infine, paiono rientrare in questa categoria della sconvenienza argomenti consolatori poco convenzionali. Nel tetrastico I89, si allude crudamente al vantaggio che la scomparsa di Cecchino può procurare ai viventi. Con la poesia I9I, invece, per sostenere l'idea confortante di sopravvivenza dell'anima dopo la morte è invocata la paura provata dai vivi nei confronti degli spiriti. La dimensione comica del corpus michelangiolesco proviene anche da una certa mancanza di misura. La mole abnorme di epitaffi che vengono scritti per un solo defunto ne è la prima manifestazione. L'altra è l'accanimento con cui la scrittura si sforza di rimanere nell'ambito di un gioco di ripetizione e variazione, unità e molteplicità. Ricorderò solo alcune emergenze di questo fenomeno che riguarda molti aspetti dei componimenti. Il cognome del defunto è oggetto di molteplici giochi onomastici. ${ }^{49} E$ frequente che una stessa coppia di parole in rima sia impiegata in più quartine (così, per « cielo-velo 》, cfr. I 88 , 209, 2I5, 227) e diversi tetrastici hanno un capoverso che inizia in modo identico (cfr. 202, 219, 225). Quasi ogni quartina esplicita $i$ tre tratti caratteristici di un epitaffio, ma la loro realizzazione concreta varia (cfr. le diverse 
formulazioni della " modalità d'esistenz̧a" : "son chiusi 》 (179), " giace» (I82), « sepulto 》 (205), « serro » (2I6), « morto sono 》 (226), ecc.) e varia pure il loro ordine d'apparizione, tanto che vengono realizzate tutte le sei possibilità combinatorie. Quanto al carattere epigrammatico delle quartine, la struttura binaria dell'epigramma può dividere $i$ quattro versi in $2+2$ (195), oppure in $3+I(I 9 I), 2^{1 / 2}+I^{1 / 2}$ (I82), ecc. Inoltre, la varietà dei tipi d'arguzia impiegati può essere ricondotta all'unità grazie alla categoria classificatoria più generale della "trasposizione", poiché le molteplici quartine sono riconducibili in sostanza alla sola tematica della consolatio. Il madrigale 192 e il sonetto 193 partecipano pure di questa nutrita dialettica fra identità e differenza. Le due poesie hanno ad esempio in comune un certo numero di parole, alcune delle quali si trovano in rima nei due componimenti, e benché i testi siano di forma metrica diversa, contano l'uno e l'altro quattordici versi.

\section{Ripetizione e esorcismo}

Vorrei concludere soffermandomi ancora in breve sull'andamento stilistico "ripetizione con variazione". Tale andamento è un forte indizio per considerare Michelangelo non soltanto in figura di consolatore, ma anche come chi cerca di esorcizzare per sé la morte di Cecchino. ${ }^{\text {so }}$

L'epitaffio del Bracci è come l'emblema della sua morte. Si può dire perciò che, scrivendo le quartine, Michelangelo ripete simbolicamente la scomparsa del giovane. La propensione a ripetere l'evento doloroso (tramite immagini, atti, ecc.) è un effetto tipico di un trauma fisico o psichico. Tuttavia, questa ripetizione è di natura ambivalente. Se da un lato manifesta la persistenza del dolore, dall' altro è un mezzo per dominare la sofferenza. Ripetere l'iscrizione tombale costituisce un modo di frammentare il trauma (e perciò di diminuirne l'impatto) e di operare su di lui, poiché lo si riproduce a volontà invece di subirlo.

Causata dal trauma e rivolta contro di lui, la ripetizione tenta di ridurlo, di annullarlo, di liberarne il soggetto. In genere, però, essa non riesce a tener fede alla sua funzione, e percio il suo compito viene sempre prorogato. La ripetizione finisce allora col perpetuarsi all'infinito. Donde ben quarantotto epitaffi composti da Michelangelo.

Franz Voelker 


\section{COMPONIMENTI FUNEBRI DI MiCHELANGELO}

i. Cito da Michelangiolo Buonarroti, Rime, a c. di E.N. Girardi, Bari, Laterza, ig6o.

2. Sui rapporti tra Michelangelo e Cecchino Bracci, cfr. R.J. Clements, The Poetry of Michelangelo, New York, New York U.P., I 965, pp. I46-48. Il Buonarroti disegnò anche la tomba del giovane e ne sorvegliò la realizzazione, ma non fu lui a eseguirla, bensì suoi collaboratori. Questa tomba murale, eretta attorno al I 546 , è tuttora visibile nell'ingresso laterale della chiesa romana di Santa Maria in Aracoeli. Cfr. C. de Tolnay, Michelangelo, Princeton, Princeton U.P., 5 voll., I 969-197 I, vol. 3, p. 8I e tavole I 24-I 25 (disegni di Michelangelo per il sepolcro) e 306 (fotografia della tomba).

3. Cfr. J.-D. Urbain, La société de conservation. Étude sémiologique des cimetières d'Occident, Paris, Payot, I978, pp. 217-I 8 .

4. Su questo punto non condivido il parere espresso da Clements, cit., p. 142, e da A.H. Hallock, Michelangelo's Revelatory Epitaphs, «Neophilologus », 67 (1983), pp. 525-39, p. 525. Secondo i due studiosi, le quartine di Michelangelo sarebbero state concepite, al momento della loro redazione, come iscrizioni potenziali, atte a venire scolpite sulla tomba di Cecchino. Per l'epigrafia rinascimentale, cfr. R. Weiss, The Renaissance Discovery of Classical Antiquity, Oxford, Basil Blackwell, I969, pp. I45-66; I. Kajanto, Classical and Christian : Studies in the Latin Epitaphs of Medieval and Renaissance Rome, Helsinki, Suomalainen Tiedeakatemia, I980; I.D. McFarlane, The Renaissance Epitaph, «The Modern Language Review », 8 I (i986), pp. XXV-XXXV. Sul valore attribuito, almeno fino al XVIII secolo, all'uso del latino nelle iscrizioni tombali, cfr. R.W. Ketton-Cremer, Lapidary Verse, "Proceedings of the British Academy », 45 (I959), pp. 237-53, pp. 239-40. Le due scritte incise sul sepolcro del giovane Bracci si leggono in Donato Giannotti, Opere politiche e letterarie, a c. di F.-L. Polidori, Firenze, Le Monnier, 2 voll., I 850 , vol. I, p. L : « D.O.M. FRANCISCO BRACCiO FLORENTINO NOBILI ADOLESCENTI IMMATVRA MORTE PRAEREPTO ANNOS AGENTI XVI DIE VIII IANVARII MDXLIV », "M.M.V. ALOYSIVS DEL RICCIO AFFINI ET ALVMNO DVLCISSIMO D. INVIDA FATA PVER MIHI TE RAPVERE SED IPSE DO TVMVLVM ET LACRYMAS QUAE DARE DEBVERAS 》.

5. La definizione scaligeriana dell'epigramma è citata e commentata in M. Blanco, Les rhétoriques de la pointe. Baltasar Gracián et le conceptisme en Europe, Paris, Champion e Genève, Slatkine, I 992, pp. I64-69. Cfr. anche P. Laurens, L'abeille dans l'ambre. Célébration de l'épigramme de l'époque alexandrine à la fin de la Renaissance, Paris, Les Belles Lettres, I989, p. Io. Il passo chiave è il seguente : «Epigramma igitur est poema breve cum simplici cuiuspiam rei vel personae vel facti indicatione aut ex propositis aliquid deducens. [...] Alia [epigrammata] vero composita sunt, quae deducunt ex propositis aliud quiddam [...]. Brevitas proprium quiddam est, argutia anima ac quasi forma ", Iulius Caesar Scaliger, Poetices libri septem. Sieben Bücher über die Dicbtkenst, a c. di L. Deitz e G. Vogt-Spira, Stuttgart-Bad Cannstatt, Frommann-Holzboog, 1994, vol. 3, p. 204.

6. Baltasar Gracián, L'acutezza e l'arte dellingegno, traduzione di G. Poggi, consulenza scientifica e coordinamento di B. Periñán, Palermo, Aesthetica edizioni, I986. Cfr. anche la sistematizzazione che di questo folto trattato barocco ha elaborato Blanco, cit., pp. 245-3 I4. A.J. Smith dedica alcune pagine alle quartine di Michelangelo in un suo libro sull'arguzia : Metaphysical Wit, Cambridge, Cambridge U.P., I991, pp. 69-7I.

7. Su Lessing, cfr. P. Laurens, cit., p. 290, Blanco, cit., pp. 195-96. Il testo della definizione dell'epigramma è il seguente, cfr. GotThOld Ephraim Lessing, Zerstreute Anmerkungen über das Epigramm und einige der vornebmsten Epigrammatisten, in Werke, a c. di H.G. Göpfert, Darmstadt, Wissenschaftliche Buchgesellschaft, 8 voll., I970-1979, vol. 5, pp. 420-529, pp. 423 e 427 : «Diese [ = die vollkommenen Sinngedichte] zerlegen sich alle von selbst in zwei Stücke; in deren einem unsere Aufmerksamkeit auf irgend einen besondern Vorwurf 


\section{FRANZ VOELKER}

rege gemacht, unsere Neugierde nach irgend einem einzeln Gegenstande gereizet wird; und in deren anderm unsere Aufmerksamkeit ihr Ziel, unsere Neugierde einen Aufschluss findet. [...] Am schicklichsten werden sich also auch die Teile des Epigramms, Erwartung und Aufschluss nennen lassen ».

8. Cfr. Laurens, cit., pp. Iо, 98 e 3 Iо, Blanco, cit., p. 247, nota I.

9. B. König, Summationsschema und Epigramm. Zerstreute Anmerkungen ₹u Ausonius (Mosella, V. 27-32) und zur lateinischen und italienischen Lyrik der Renaissance, in Ausonius, a c. di M.J. Lossau, Darmstadt, Wissenschaftliche Buchgesellschaft, I99I, pp. 20I-28, pp. 219-20.

io. Su Luigi del Riccio, cfr. Michelagniolo Buonarroti, Die Dicbtungen, a c. di C. Frey, Berlin, Walter de Gruyter \& Co., 1964², pp. 528-29; E. Steinmann, Michelangelo e Luigi del Riccio, Firenze, Vallecchi, I932; P. Procaccioli, voce "Del Riccio, Luigi», in Dirionario Biografico degli Italiani, Roma, Istituto della Enciclopedia Italiana, vol. 38, I990, pp. 263-65.

i i. Le annotazioni di Michelangelo si leggono in Buonarroti, ed. Girardi, cit., pp. 368-79.

I 2. Questa variante che evoca il letto e gli abbracci è stata omessa, ossia censurata nella prima edizione moderna delle Rime (Le rime di Michelangelo Buonarroti, pittore, scultore e arcbitetto, a c. di C. Guasti, Firenze, Le Monnier, I863, p. Iо, n. I7). Viene commentata da G. Gorni, Obscurité et transparence dans les poèmes de Michel-Ange, "Cahiers de la Faculté des Lettres de l’Université de Genève ", 4/I (I99I), pp. I3-I9, p. i 5.

I 3. Sul Codice Magliabechiano VIII. 38, cfr. Buonarroti, ed. Frey, cit., p. 298, ed. Girardi, cit., pp. 498-99. I testi non buonarrotiani di questa collettanea per Cecchino, rimasta manoscritta, si leggono in Buonarroti, ed. Frey, cit., pp. 267-71.

14. Cfr. A. Fleges, «Je ravie le mort». Tombeaux littéraires en France à la Renaissance, «La licorne », 29 Le tombeau poétique en France (1994), pp. 7 1-I42, pp. I08-I 2. Va osservato che il poscritto al madrigale 192 fornisce un esempio dell'interesse di Michelangelo per la produzione di un suo «concorrente».

i 5. Cfr. Buonarroti, ed. Frey, cit., p. 359.

16. Un dispositivo analogo (un gioco onomastico serve allora da legame fra i testi) è costituito dalla quartina 177 e dal sonetto 178 . Nel verso «se l'un ne l'altro amante si trasforma» (193, I 2 e 194, 4) riecheggia un passo petrarchesco, Triumphus Cupidinis, III, I6I-I62: «e so in qual guisa / l'amante ne l'amato si trasforme». Questa trasformazione dell'amante nella persona che ama è un motivo tradizionale che trova ampi sviluppi nell'ambito del neoplatonismo fiorentino, cfr. G. Cambon, La poesia di Michelangelo. Furia della figura, Torino, Einaudi, I991, p. 173, e Michelangelo, Rime, a c. di M. Residori, Milano, Mondadori, I998, p. 3 I 9 .

17. Cfr. L. Ghizzoni, Indagine sul « canzoniere» di Michelangelo, «Studi di filologia italiana », 49 (1991), pp. 167-87.

i 8. Donato Giannotti, Dialogi de' giorni che Dante consumò nel cercare l'Inferno e 'l Purgatorio, a c. di D. Redig de Campos, Firenze, Sansoni, 1939, pp. 70 e 98.

I 9. Il testo della Lezione del Varchi si legge in Le rime di Michelangelo Buonarroti, ed. Guasti, cit., pp. LXXXV-CXII, ripreso da Due Lezzioni di M. Benedetto Varchi, nella prima delle quali si dichiara un Sonetto di M. Michelagnolo Buonarroti. Nella seconda si disputa quale sia più nobile arte la Scultura, o la Pittura; con una Lettera d'esso Michelagnolo, et più altri Eccellentiss. Pittori, et Scultori, 


\section{COMPONIMENTI FUNEBRI DI MiCHELANGELO}

sopra la Quistione sopradetta, In Fiorenza. Appresso Lorenzo Torrentino Impressor Ducale. MDXLIX.

20. Giorgio VAsari, Le vite de’ piú eccellenti architetti, pittori, et scultori italiani, da Cimabue insino a' tempi nostri nell'edizione per i tipi di Lorenzo Torrentino. Firenze I550, a c. di L. Bellosi e A. Rossi, Torino, Einaudi, I986, p. 903. La quartina sarebbe stata scritta da Michelangelo fra la fine del i 545 e il principio del i 546 (cfr. Buonarroti, ed. Girardi, cit., p. 403).

2. Sul legame tra il genere dell'epigramma e una scrittura del tipo "variazione sul tema ", cfr. Laurens, cit., pp. $65-96$.

22. Cfr. S. Carrai, Machiavelli e la tradizione dell'epitaffio satirico fra Quattro e Cinquecento, «Interpres ", 6 (1985-1986), pp. 200-13.

23. Questi testi si leggono in Epigrammi italiani, a c. di G. Mazzoni, Firenze, Barbèra, i 896, nn. $955-970$, pp. 3 II-I6.

24. Cfr. M. Plaisance, Une première affirmation de la politique culturelle de Côme $I^{e r}$ : la transformation de l'Académie des "Humidi en Académie florentine (I540-I542), in Les écrivains et le pouvoir en Italie à l'époque de la Renaissance (première série), a c. di A. Rochon, Paris, Université de la Sorbonne nouvelle, 1973, pp. 361-438, p. 395. Interessante rilevare che gli statuti dell'Accademia degli Umidi prevedevano la composizione di «Epitaffi o madrigali o sonetti » e la loro diffusione al di fuori della cerchia dei membri, cfr. Plaisance, cit., pp. 388-89 e la voce "Accademia degli Umidi» in M. Maylender, Storia delle accademie d'Italia, Bologna, Arnoldo Forni, 5 voll., I 976 (ristampa della I ed., Bologna, Capelli, I926-1930), vol. 5, pp. 363-67, pp. 364-65.

25. Cfr. Anton Francesco Grazzini, Le rime burlesche, edite e inedite, a c. di C. Verzone, Firenze, Sansoni, I 882, pp. 635-41, sezione «Epitaffi».

26. Pasquinate del Cinque e Seicento, a c. di V. Marucci, Roma, Salerno, 1988, n. CXV, pp. I 53-54, serie di sette quartine del I 541-1 542 ; n. CXXIII, pp. I65-73, serie di trentotto quartine del I 544

27. Si annoverano nelle Vite una ventina di quartine a rime incrociate : cfr. VASARI, cit., pp. 235, 240, 259, 273, 326, 333, 387, 401, 503, 508, 570, 586, 663, 695, 757, 785, 805 . Su questi epitaffi e i problemi relativi alla loro redazione (autore, datazione), cfr. R. Bettarini, Vasari scrittore : come la Torrentiniana diventò Giuntina, in Il Vasari storiografo e artista. Atti del congresso internazionale nel IV centenario della morte (Arez:o-Firenze, 2-8 settembre 1974), Firenze, Istituto Nazionale di Studi sul Rinascimento, 1976, pp. 485-500; Giorgio Vasari, Catalogo delle mostre tenutesi a Arezzo dal 26 settembre al 29 novembre I98 I, Firenze, EDAM, I981, pp. 219-20; VASARI, cit., pp. XXXVII-XXXVIII.

28. Tra le quartine di Michelangelo che esprimono in primo luogo il dolore di chi perde Cecchino vanno menzionati i tetrastici 207, 210, 2 II.

29. La lettera è citata in Buonarroti, ed. Frey, cit., p. 532.

30. Una divisione degli argomenti consolatori in tre classi è indicata da Cicerone, Tusculanae Disputationes, III, xxxII, 77, citato anche da E. Safty, Les sources grecques et latines des principaux topoi des consolations contre la mort dans la poésie baroque, «Papers on French Seventeenth Century Literature », vol. XXIII, n. 44 (I996), pp. 303-28, p. 303 : «Erit igitur in consolationibus prima medicina docere aut nullum malum esse aut admodum parvum, altera et de communi condicione vitae et proprie, si quid sit de ipsius qui maereat dispu- 


\section{FRANZ VOELKER}

tandum, tertia summam esse stultitiam frustra confici maerore, cum intellegas nihil posse profici». A guidarmi sono i seguenti repertori tematici : per la consolatio classica, B. Lier, Topica carminum sepulcralium latinorum, "Philologus», 62-16 (1903), pp. 445-77, 563-603 e 63-17 (1904), pp. 54-65; R. Lattimore, Themes in Greek, and Latin Epitaphs, Urbana, University of Illinois Press, 1962; per la consolatio cristiana, P. von Moos, Consolatio. Studien zur mittellateinischen Trostliteratur über den Tod und zum Problem der cbristlichen Trauer, München, Wilhelm Fink, 4 voll., 1972.

31. La personificazione della morte con tratti quali la crudeltà $(185,2)$, l'ingordigia $(200,3)$, l'invidia $(2$ I 2, 4) serve a ritrarre questa sua ineluttabilità.

32. Lier, cit., \22, pp. 569-71, « De pugna contra Mortem inutili ». È facile incontrare negli epitaffi cinquecenteschi in volgare, siano comici oppure seri, un gioco sul nome del morto. Nel VaSARI, cit., si legge in una delle iscrizioni tombali che chiudono la sezione dedicata a Leonardo da Vinci : «VINCE COSTVI PVR SOLO / TVTTI ALTRI; E VINCE FIDIA, E VINCE APELLE, / E TVTTO IL LOR VITTORIOSO STVOLO » (p. 556 ). L'epitaffio che conclude la vita di Morto da Feltre comincia con il verso seguente : « MORTE HA MORTO NON ME CHE IL MORTO SONO» (p. 785 ). Giochi onomastici simili si possono trovare pure fra le scritte funerarie antiche. A chi sfoglia un'antologia d'iscrizioni tombali romane capita presto di leggere : "Felix vocatus, felix vixit cum suis » (tomba d'Alfius Flavianus Felix), cfr. Tombeaux romains. Anthologie d'épitaphes latines, a c. di D. Porte, Paris, Gallimard, I993, p. 20. Una lista d'esempi affini è data da A. Brelich, Aspetti della morte nelle iscrizioni sepolcrali dell'impero romano, Budapest, Istituto di Numismatica e di Archeologia dell'Università, 1937, p. 46. Nel suo articolo, Les éléments figuratifs des "Carmina Latina Epigraphica", in Anamnesis. Gedenkboek Prof. Dr. E.A. Leemans, Brugge, De Tempel, 1970, pp. 317-41, p. 322, G.M. Sanders afferma che le allusioni al "contenuto" del nome proprio sono un fatto corrente nella mentalità antica. Perciò, a motivare su un piano letterario il gioco onomastico nell'epitaffio volgare cinquecentesco potrebbe essere anche l'imitazione di una pratica frequente nelle scritte funerarie dell'antichità. Altri valori, di natura antropologica, sono pure possibili per i giochi onomastici in un contesto funebre (cfr. Brelich, cit., pp. 32-53). Nel caso del Bracci, va anche precisato che il sepolcro del giovane in Santa Maria in Aracoeli ostenta le armi di famiglia ai due lati, che riproducono l'immagine di un braccio ripiegato.

33. Su questo "pericolo della longevità " (« Gefahr der Langlebigkeit »), cfr. von Moos, cit., vol. 3, SS 784-81 5, pp. I64-72.

34. Francesco Petrarca, Familiarium rerum libri, II, i, 2, citato da G.W. McClure, Sorrow and Consolation in Italian Humanism, Princeton (New Jersey), Princeton U.P., I991, p. 3 I : " consolatio enim, ut in mesti auditoris animum penetret, eget multa verborum maiestate, eget gravibus animosisque sententiis ».

35. Cfr. Blanco, cit., pp. 275-79 per l'arguzia del tipo «sentenza », pp. $254^{-5} 8$ per quella del tipo « correlazione».

36. La similitudine delle finalità perseguite dall'arguzia e dalla consolatio appare se si ravvicina Blanco, cit., pp. 267, 286 (sull'arguzia) e Fleges, cit., pp. I 23-24 (sul discorso consolatorio). Fleges scrive in particolare (suoi i corsivi) : «Plus généralement, l'emprise de l'idéologie se traduit par une économie généralisée du sens : pas de mort qui n’ait [...] une possible explication. [...] C'est ramener [...] l'inconnu (l'injustifiable intrusion de la mort) à du connu, l'indicible à du formulable. Tout, dans un discours aussi pénétré d'idéologie que celui du Tombeau, doit faire sens. La mort surtout, dont il faut sans cesse justifier le scandale, formaliser les opérations. D'où le recours constant au même corpus de fictions : jalousie (ou faveur) des dieux, manifestation de leur colère [...]. Le recours au topos a là encore pour fonction de produire du sens; on serait tenté de dire : faire en sorte de ne rien 


\section{COMPONIMENTI FUNEBRI DI MiCHELANGELO}

laisser au hasard. Peu importe le degré de vraisemblance de la fiction. L'ESSENTIEL EST DE CONVERTIR L'ÉVÉNEMENT EN SIGNE. [...] Le Tombeau fonctionne comme une machine à produire du sens [...]» (p. I 24). E Blanco : «Comme dans toutes les espèces de trait vues jusqu'ici, on voit, dans cet exemple de comparaison conditionnelle, que l'appel à un contexte éloigné ISOLE UN FRAGMENT DE RÉCIT, UN ÉLÉMENT D’ANECDOTE ET LUI DONNE LE STATUt D'Un SIgNe. La pointe par correspondance peut faire de la naissance de Saint Jean Baptiste un signifiant de sa mort, et de sa mort un signifiant de sa naissance» (p. 267).

37. Blanco, cit., pp. 268-72; Gracián, cit., Discorso XVII, Delle ingegnose trasposiz̨ioni, pp. I 35-36 : «Questa specie di concetti è una delle più gradevoli che sia dato d'osservare. La sua tecnica consiste nel trasformare l'assunto convertendolo nel contrario di ciò che appare [...]. La prontezza ingegnosa consiste nel commentare l'infortunio trasformandolo in fortuna e facendolo apparire come un'occasione propizia ».

38. Urbain, cit., pp. I42-43; cfr. anche Fleges, cit., p. 92.

39. G. Gorni, Metrica e analisi letteraria, Bologna, il Mulino, I993, p. I 29.

40. Lier, cit., $\int 28$, pp. 578-83, «Vita a natura mutua data est»; von Moos, cit., vol. 3,

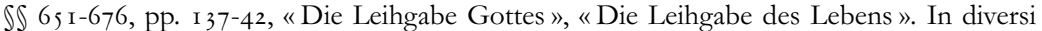
testi citati da von Moos, la parola latina «creditor» serve a designare la posizione di Dio rispetto all'uomo.

4I. Vari poscritti di Michelangelo qualificano certe quartine con il termine di «goffo » (cfr. le annotazioni sotto i tetrastici I94, I99, 2 I I e 2 I4). L'espressione potrebbe riguardare il tenore dei testi e significare "insulso", "assurdo". Se invece l'epiteto si applica alla fattura delle poesie, il suo senso sarebbe quello di "sgraziato", " artisticamente non riuscito". "Goffo » figura sotto le quartine 2 I I e 2 I 4 assieme alla menzione della fretta che presiede alla scrittura. Mi sembra perciò, come propone A.J. Smith, For the Death of Cecchino Bracci, «The Modern Language Review», 58 (1963), pp. 355-63, p. 361, che " goffo » si riferisca alla qualità estetica delle poesie e non all'uso di argomenti consolatori che vi viene fatto, argomenti il cui valore non dipende dal tempo dedicato a comporre i versi. Sempre secondo Smith (p. 363), « goffo » avrebbe, come la maggioranza dei poscritti, una funzione di autodifesa : conscio di non essere un letterato, un uomo del mestiere, il Buonarroti denigrerebbe in un modo o nell'altro la propria produzione per smorzare le possibili critiche degli altri partecipanti alla collettanea. Non sono convinto che i poscritti avessero un carattere pubblico. Sembrano soprattutto, se non esclusivamente, destinati a Luigi del Riccio. La variante «morale» della quartina 197 , le richieste fatte a Luigi affinché egli « racconci » a suo modo alcuni testi buonarrotiani (cfr. i commenti sotto I93 e 208) paiono indirizzarsi solo all'amico, a un amico per giunta benevolo. A riprova della natura privata delle annotazioni, ricorderò che Luigi del Riccio non le ha ricopiate nella collettanea. Ad ogni modo, considerare, come Smith, che l'insieme dei commenti svolge essenzialmente una funzione di autodifesa ne riduce, a parer mio, eccessivamente le potenzialità. Va segnalato infine che Fleges, cit., pp. I 24-25, incontra nel suo corpus di collettanee in morte dei casi di contestazione di argomenti consolatori.

42. McClure, cit., pp. 78-80 (a proposito di Coluccio Salutati).

43. S. Longhi, Lusus. Il capitolo burlesco nel Cinquecento, Padova, Antenore, I983, p. 217 : «l'identità della poesia burlesca risiede nella sua mancanza di convenienza e di misura, nelle sue deroghe alla norma per eccesso o per difetto ».

44. Cfr. D. Romei, «Bernismo» di Michelangelo, in Berni e berneschi del Cinquecento, Firenze, Centro $2 \mathrm{P}$, 1984, pp. $137-82$. 


\section{FRANZ VOELKER}

45. R. e M. Wittkower, Les enfants de Saturne. Psychologie et comportement des artistes, de l'Antiquité à la Révolution française, traduzione di D. Arasse, Paris, Macula, I991, pp. 39-40.

46. P. Orvieto, Pulci medievale. Studio sulla poesia volgare fiorentina del Quattrocento, Roma, Salerno, I 978 , p. 195 .

47. La prima attestazione di « finocchio » nel senso di «omosessuale maschile» risalirebbe alla fine del Duecento, ma «data l'oscurità del brano e il forte iato cronologico tra questa attestazione e la seconda (I 863) », il fatto è controverso (M. Cortelazzo, P. Zolli, DELIDižionario etimologico della lingua italiana, a cura di M. Cortelazzo e M.A. Cortelazzo, Bologna, Zanichelli, $1999^{2}$, p. 587 ).

48. La quartina 2 I6 ravvicina «il Braccio», ossia Cecchino, a un «bel coltello» nella « vagina ». Ora, nel «linguaggio dell'equivoco », tipico della poesia burlesca del Cinquecento «braccio » e « coltello » acquistano il senso di «membro virile», cfr. G. Ferroni, Il doppio senso erotico nei canti carnascialeschi fiorentini, "Sigma», I I (1978), pp. 233-50, e J. Toscan, Le carnaval du langage. Le lexique érotique des poètes de l'équivoque de Burchiello à Marino $\left(X V^{e}\right.$-XVII ${ }^{e}$ siècles), Lille, Atelier Reproduction des thèses Université de Lille III, 4 voll., I 98 I. Il tetrastico 222, dal canto suo, presenta la triade « acerbo-frutto-cede » e Cecchino vi viene "goduto » e "posseduto » dalla tomba. La sensualità di queste varie espressioni è segnalata da S. Matarasso-Gervais nella premessa (p. 63) e nel commento (p. I 54 ) alla sua traduzione di Michel-Ange Buonarroti, Épitaphes pour la mort de Francois des Bras, Aix-enProvence, Alinea, 1983. Questa sensualità velata appartiene, direi, a un altro registro che quello comico. Il sesso e la morte, si sa bene, sono connessi l'uno all'altro.

49. Cfr. I 84, I : i « bracci » sono deboli ; I 97, 4 (variante) : Cechino «ab-bracc[i]-ava » ; 2 I 2 , I-2 : il «braccio » non aveva pari per ferir altrui ; 2 I $3, \mathrm{I}-2:$ il «braccio » doveva servire a « corregger [...] la natura »; 222, I-2 : la morte «stese il Braccio », ossia « distese il corpo di Cecchino Bracci », ma anche « allungò il braccio », cfr. MicheLAngeLo, ed. Residori, cit., e Michelangelo, Rime e lettere, a cura di P. Mastrocola, Torino, UTET, I992. Questi giochi sul nome hanno probabilmente una valenza comica. Altre funzioni sono tuttavia possibili (cfr. più sopra nota 32 ).

50. Cfr. M. Collot, Exorcisme et répétition, in La matière-émotion, Paris, Presses Universitaires de France, 1997, pp. I60-85, il quale si ispira a proposito di testi di Henri Michaux al concetto freudiano di "coazione a ripetere" («Wiederholungszwang»). Nella collettanea per Cecchino, un componimento di Carlo Gondi associa Luigi del Riccio e Michelangelo nel dolore per la scomparsa del giovane : «Morte [...] / Per gelosia del Riccio et per far' guerra / Al Buonarroti [...] / [...] lo levò di terra ; / Onde d'un colpo fu di tre rapace : / Di lui qui il corpo e de i duo' l'alma iace», (cfr. Buonarroti, ed. Frey, cit. p. 270, n. io). 